
adolescentes do sexo feminino. Cuadernos de Psicología del Deporte, Vol 19(2), 256-264

\title{
Relação entre antropometría, gordura corporal e autoconceito de adolescentes do sexo feminino
}

\section{Relationship between anthropometry, body fat and self concept of femaly sex adolescents}

\section{Relación entre antropometría, gordura corporal y autoconceito de adolescentes del sexo femenino.}

\author{
Falconi, C.A. ${ }^{1,2}$; Santos, T. A. ${ }^{1,2}$; Figueira Junior, A. J. ${ }^{1,2}$; Neves, A. N. ${ }^{4}$; Andrade, E. L. ${ }^{5}$; Ferreira \\ Brandão, M. R. F. ${ }^{1,2}$; Zanetti, M. C. ${ }^{1,2,3}$.
}

${ }^{1}$ Universidade São Judas Tadeu; ${ }^{2}$ Grupo de Estudos em Psicofisiologia e Treinamento Aplicados ao Esporte e Exercício - GEPTAEE; ${ }^{3}$ UNIP -Universidade Paulista- São José do Rio Pardo ${ }^{4}$ Escola de Educação Física do Exército do Rio de Janeiro; ${ }^{5}$ Universidade Nove de Julho.

\begin{abstract}
RESUMO
O excesso de peso causado pelo acúmulo de gordura corporal e a circunferência de cintura aumentada podem ocasionar riscos à saúde dos jovens, contribuindo para o desenvolvimento de problemas psicossociais precoces e redução da qualidade de vida do adolescente. Nesse contexto, o autoconceito é um importante indicador de bemestar psicológico, principalmente na idade jovem. Dentre os inúmeros fatores que podem contribuir para a percepção do autoconceito está a composição corporal, que é constantemente modificada nessa fase da vida. Nesse sentido, o acúmulo excessivo de gordura corporal e o aumento da circunferência abdominal podem estar associadas a autoconceitos positivos ou negativos sobre si mesmo, favorecendo o desenvolvimento de fatores psicossociais da população jovem em prol de comportamentos saudáveis ou não. Assim, esse trabalho buscou analisar a relação da circunferência de cintura, o percentual de gordura e o autoconceito físico e global de adolescentes do sexo feminino. Para isso, foi realizado um estudo transversal com a participação de 101 adolescentes do sexo feminino, estudantes do Ensino Médio de uma Escola Técnica Estadual da cidade de São Paulo. Foi utilizada a escala de Autopercepção de Harter para Adolescentes validada para adolescentes Brasileiros, nas dimensões do autoconceito físico e global, e aferidas medidas antropométricas de massa corporal e estatura para a verificação do IMC (índice de massa corporal), pregas cutâneas para o percentual de gordura e perímetros para a circunferência de cintura. Foi encontrada uma correlação pequena e inversa entre as variáveis autoconceito físico e circunferência de cintura corroborando com outros estudos que apontaram resultados similares.
\end{abstract}




\section{Falconi, C.A.; Santos, T. A., Figueira Junior, A. J., Neves, A. N., Andrade, E. L. ; Brandão, M. R. F.; Zanetti, M. C.}

Palavras chave: autoconceito; autoconceito físico; adolescente; antropometria.

\section{RESUMEN}

El exceso de peso causado por la acumulación de grasa corporal y la circunferencia de cintura aumentada pueden ocasionar riesgos para la salud de los jóvenes, contribuyendo al desarrollo de problemas psicosociales precoces y reducción de la calidad de vida del adolescente. En ese contexto, el autoconcepto es un importante indicador de bienestar psicológico, principalmente en la edad joven. Entre los innumerables factores que pueden contribuir a la percepción del autoconcepto está la composición corporal, que es constantemente modificada en esa fase de la vida. En este sentido, la acumulación excesiva de grasa corporal y el aumento de la circunferencia abdominal pueden estar asociadas a autoconceptos positivos o negativos sobre sí mismo, favoreciendo el desarrollo de factores psicosociales de la población joven en pro de comportamientos sanos o no. Así, ese trabajo buscó analizar la relación de la circunferencia de cintura, el porcentaje de grasa y el autoconcepto físico y global de adolescentes del sexo femenino. Para ello, se realizó un estudio transversal con la participación de 101 adolescentes del sexo femenino, estudiantes de la Enseñanza Media de una Escuela Técnica Estadual de la ciudad de São Paulo. Se utilizó la escala de Autopercepción de Harter para Adolescentes validada para adolescentes brasileños, en las dimensiones del autoconcepto físico y global, y medidas medidas antropométricas de masa corporal y estatura para la verificación del IMC (índice de masa corporal), pliegues cutáneos para el porcentual de masa corporal grasa y perímetros para la circunferencia de cintura. Se encontró una correlación pequeña e inversa entre las variables autoconcepto físico y circunferencia de cintura corroborando con otros estudios que apuntar resultados similares.

Palabras clave: autoconcepto; autoconcepto físico; adolescente; antropometría.

\section{ABSTRACT}

Excess weight caused by accumulation of body fat and increased waist circumference can cause health risks for young people, contributing to the development of early psychosocial problems and reduced quality of life of adolescents. In this context, self-concept is an important indicator of psychological well-being, especially in young age. Among the many factors that may contribute to the perception of self-concept is the body composition, which is constantly modified in this phase of life. In this sense, excessive accumulation of body fat and increased abdominal circumference may be associated with positive or negative self-concepts, favoring the development of psychosocial factors in the young population for healthy behaviors. Thus, this study sought to analyze the relationship of waist circumference, fat percentage and the physical and global self-concept of female adolescents. For that, a cross-sectional study was carried out with the participation of 101 female adolescents, students of the High School of a State Technical School of the city of São Paulo. The Harter self-perception scale for adolescents was validated for Brazilian adolescents, in the dimensions of physical and global self-concept, and anthropometric measures of body mass and height were checked for BMI (body mass index), skin folds for the percentage of fat and perimeters for waist circumference. A small inverse correlation was found between the variables physical selfconcept and waist circumference corroborating with other studies that indicated similar results.

Keywords: self-concept; physical self-concept; adolescent; anthropometry.

\section{INTRODUÇÃO}

A obesidade tem sido um dos problemas de saúde pública mais alarmantes nos países desenvolvidos e em desenvolvimento (WHO, 2006). O balanço energético positivo é resultado de dois fatores comportamentais, mantidos por período prolongado: a alimentação hipercalórica e o comportamento sedentário (WHO, 2006; SBH, 2005). Durante a adolescência, o jovem passa por profundas mudanças físicas, psicológicas e sociais, incidindo em novos comportamentos e responsabilidades que contribuirão para a transição da fase de dependência para a de independência (Kvaavic et al., 2009). O excesso de peso gera aumento da gordura visceral podendo favorecer o desenvolvimento de doenças metabólicas futuras (IDF, 2007; WHO, 2006; SBH, 2005), mas de imediato pode afetar o bem-estar psicossocial do adolescente, sobretudo do sexo feminino (Matsudo,

Cuadernos de Psicología del Deporte, 19, 2 (mayo) 


\section{Medidas do corpo e autoconceito de adolescentes do sexo feminino}

2017; DBO, 2016; Guerra et al., 2016; Pereira, 2016; Massuça \& Proença, 2013; Santana et al. 2013).

A interação social proporcionada pela convivência entre os pares influência o conceito abstrato que o adolescente cria sobre si, por meio dos feedbacks das pessoas que o jovem atribui significância (professores, amigos e pais), e também acerca das experiências dessa convivência (Gárcia-Sanchés, 2013; Jelalian et al., 2011; Adams \& Bukowski, 2008; Villa Sánches \& Escribano, 1999). Nesse sentido, o autoconceito é definido como a percepção que o indivíduo tem a respeito de suas competências, incluindo aspectos físicos, acadêmicos e sociais (Bandeira, Arteche e Reppold, 2008; Santana, 2003), possibilitando a completa diferenciação de si em relação ao meio (Bee, 1996). Nessa fase da vida, o adolescente busca se associar à grupos de identificação para fortalecer o sentimento de autovalorização, a caracterização de sua identidade e a internalização do seu autoconceito diante das diversas formas de interação com o meio, seja como aluno, filho, amigo, namorado e atleta, dentre outros (Harter, 1999; Villa Sánches; Escribano, 1999).

O termo autoconceito apresenta certas divergências em relação a sua definição e diferenciação quanto a autoestima (Monteiro, 2012; Bandeira, Arteche e Reppold, 2008). A autoestima é a atribuição de valor que o indivíduo faz sobre si, de forma afetiva, unidimensional e geral (Monteiro, 2012). García e Gómez (2010) consideram que a autoestima depende do autoconceito que o indivíduo tem nos diferentes domínios (físico, social, acadêmico, atlético dentre outros), já que ela parte de uma análise afetiva para estabelecer a autoestima da pessoa, de forma geral. $\mathrm{O}$ autoconceito é mais amplo e multidimensional e o mesmo indivíduo pode ter autoconceito alto ou baixo em diferentes domínios (García; Gómez, 2010).

Nesse sentido, a partir da fase da puberdade, as adolescentes valorizam a aparência física $\mathrm{e}$ constroem o conceito de serem ou não serem bonitas (Gárcia-Sanchés, 2013; Jelalian et al., 2011; Kvaavic et al. 2009; Adams \& Bukowski, 2008). A aparência física considera aspectos como altura, massa corporal e sua distribuição para criar uma representação mental da pessoa sobre seu próprio corpo. Então, no domínio do autoconceito físico, a aparência física é avaliada pelo do grau de satisfação do indivíduo com seu corpo, se o indivíduo se considera bonito. No mais, o autoconceito pode ser expresso como uma avaliação global, que é representada por "um julgamento global ao invés de uma avaliação por domínios", ele representa a satisfação do indivíduo consigo próprio, mostra se a pessoa, em geral, é feliz (Bandeira, Arteche e Reppold, 2008, p. 85). Assim, o objetivo desse estudo foi analisar a relação entre as medidas antropométricas, o percentual de gordura e o autoconceito físico e global de adolescentes do sexo feminino.

\section{MATERIAL E MÉTODOS}

\section{Participantes}

Foram investigados 101 adolescentes do sexo feminino, estudantes do ensino médio de uma Escola Técnica Estadual, com idade entre 15 e 17 anos, localizada na região leste da cidade de São Paulo. A instituição tem 360 adolescentes matriculados nesse grau de ensino. Todas as participantes eram alfabetizadas e sem limitações cognitivas.

\section{Instrumentos}

$\mathrm{O}$ autoconceito das participantes foi mensurado pela versão da Escala de Autopercepção de Harter para Adolescentes brasileiros (alfa de Cronbach 0,60 a $0,88)$. Embora essa escala apresente nove domínios diferentes, para esse estudo, somente os domínios do autoconceito físico e autoconceito global foram utilizados (Bandeira, 2008).

Para a classificação do IMC- (índice de massa corporal) dos participantes foram utilizadas as curvas da Organização Mundial da Saúde - OMS (2007), ajustadas para sexo e idade. Para o cálculo do percentual de gordura foi aplicada a equação de Slaughter et al. (1988). A referência da circunferência de cintura seguiu a recomendação do IDF (2007), que considera que $\leq 80 \mathrm{~cm}$ é o limite de normalidade considerando uma boa saúde para adolescentes do sexo feminino.

\section{Procedimentos}

Esse estudo foi aprovado pelo Comitê de Ética em Pesquisa da Universidade São Judas Tadeu, sob o registro $\mathrm{n}^{\circ}$. 118072/2017. E está de acordo com a Declaração de Helsinque, conforme revisão de outubro de 2000. As participantes tiveram contato 
Falconi, C.A.; Santos, T. A., Figueira Junior, A. J., Neves, A. N., Andrade, E. L. ; Brandão, M. R. F.; Zanetti, M. C.

com a pesquisa durante uma palestra realizada na própria escola, ministrada aos responsáveis das adolescentes abordando os objetivos e a importância acadêmica do estudo. Os responsáveis pelas participantes assinaram o Termo de Consentimento Livre e Esclarecido, e as adolescentes assinaram, o Termo de Assentimento demostrando ciência e concordando em participar de todas as etapas da pesquisa. As adolescentes que cumpriram com os aspectos legais de sua participação foram orientadas quanto a data da coleta. Após a orientação quanto ao preenchimento da escala de autoconceito, as adolescentes a preencheram de forma individual, acomodadas em cadeiras e mesas individuais, em uma sala de aula da própria instituição. As participantes também foram instruídas a não conversassem com as demais colegas até o término do preenchimento. As dúvidas em relação a compreensão das questões foram individualmente esclarecidas pelos aplicadores. A estação de coleta das variáveis antropométrica foi montada em outra sala da escola para garantir privacidade às participantes, que eram conduzidas até o local de coleta após o preenchimento da escala. As participantes estavam vestindo roupas leves, com camiseta, bermuda ou shorts e sem tênis, conforme a orientação da equipe de coleta.

As medidas antropométricas incluíram: massa corporal, aferida com a balança digital HN-289 Balança Digital Corporal - Omron, com capacidade para $150 \mathrm{~kg}$ e precisão $0,01 \mathrm{Kg}$; estatura, medida com estadiômetro de alumínio da marca Sanny com precisão de $0,1 \mathrm{~cm}$; circunferência de cintura, medida por uma fita métrica metálica de 2 metros com precisão de $0,1 \mathrm{~cm}$, no ponto médio entre o último arco costal e a crista ilíaca. Para garantir a fidedignidade das medidas todas as variáveis foram repetidas por 3 vezes e calculada a média. Para calcular o percentual de gordura corporal foram medidas as pregas cutâneas tricipital e geminal usando o adipômetro científico Prime Med. A30 de Aço, modelo A30, com classificação científica 0,1 $\mathrm{cm}$.

\section{Tratamento Estatístico}

Para o tratamento dos dados utilizou-se o pacote estatístico SPSS na versão 21.0 para Windows. Foram realizadas análises descritivas e testes de normalidade. Para as variáveis que se ajustaram à normalidade foram feitas análises paramétricas com o teste $\mathrm{t}$ de Student para grupos independentes, divididas pela circunferência abdominal. Para as variáveis que não se ajustaram a normalidade, foram feitas análises não paramétricas pelo teste de correlação de Spearman (Field, 2009). Para todas as análises, foi adotado o nível de significância de $\mathrm{p}<0,05$.

\section{Resultados}

Na tabela 1 são apresentados os valores de média e desvio padrão das adolescentes. A faixa etária das participantes variou entre 15,1 e 18,6 anos, com circunferência de cintura e percentual de gordura corporal apresentando valores médios abaixo da classificação de risco para o desenvolvimento de doenças metabólicas, sendo a primeira $\leq 80 \mathrm{~cm}$ (IDF, 2007) e a segunda $\leq 30$ (Lohman et al., 1992).

Tabela 1.

Média e desvio padrão das variáveis do autoconceito e do estado nutricional das adolescentes.

\begin{tabular}{|c|c|c|c|}
\hline Variáveis & Média & & DP \\
\hline Idade & 16,60 & \pm & 0,89 \\
\hline IMC - Índice de massa corporal & 21,20 & \pm & 3,57 \\
\hline Circunferência abdominal & 75,12 & \pm & 8,85 \\
\hline Percentual de gordura & 25,07 & \pm & 6,96 \\
\hline Autoconceito físico & 2,19 & \pm & 0,87 \\
\hline Autoconceito global & 2,53 & \pm & 0,86 \\
\hline
\end{tabular}

Cuadernos de Psicología del Deporte, 19, 2 (mayo) 


\section{Medidas do corpo e autoconceito de adolescentes do sexo feminino}

A distribuição das participantes é apresentada na tabela 2, mostrando que $72,3 \%$ das adolescentes apresentavam-se na classificação do IMC por percentil como eutrófico, enquanto $21,8 \%$ estavam na faixa de excesso de peso para a estatura (WHO,
2007). Além disso, também $72,3 \%$ da amostra apresentou circunferência de cintura dentro dos parâmetros de normalidade, conforme considera o IDF (2007).

Tabela 2.

Caracterização dos participantes do estudo conforme a idade, o IMC e a circunferência abdominal, apresentados por frequência e porcentagem.

\begin{tabular}{ccc}
\hline Variável & Classificação & n. (\%) \\
\hline & 15 anos & $32(31,7)$ \\
& 16 anos & $29(28,7)$ \\
Idade & 17 anos & $36(35,6)$ \\
& 18 anos & $4(4,0)$ \\
& Total & $101(100)$ \\
\hline & Baixo Peso & $6(5,9)$ \\
IMC & Eutrófico & $73(72,3)$ \\
& Sobrepeso & $18(17,8)$ \\
& Obesidade & $4(4,0)$ \\
& Total & $101(100)$ \\
\hline Circunferência & $\leq 80 \mathrm{~cm}$ & $73(72,3)$ \\
Cintura & $\geq 80,1 \mathrm{~cm}$ & $28(27,7)$ \\
& Total & $101(100)$ \\
\hline
\end{tabular}

IMC = índice de massa corporal.

$\mathrm{Na}$ tabela 3, são apresentadas as comparações das médias dos grupos divididos pela circunferência de cintura. As adolescentes com valores iguais ou menores que 80 centímetros alcançaram significativamente maior pontuação na escala de autoconceito físico em comparação ao grupo com circunferência maior que este valor. Já o autoconceito global não apresentou médias significativamente diferentes quando comparados por essa mesma variável.

$\mathrm{T}$

\section{Tabela 3.}

Comparação das médias do grupo dividido pela medida de circunferência abdominal.

\begin{tabular}{lccccc}
\hline \multicolumn{1}{c}{ Variável } & $\begin{array}{c}\leq 80 \mathrm{~cm} \\
\mathbf{n . 7 3}\end{array}$ & $\begin{array}{c}\geq 80,1 \mathrm{~cm} \\
\mathbf{n . 2 8}\end{array}$ & $\boldsymbol{t}$ & $\boldsymbol{p}$ & $\mathbf{9 5 \% ~ I C}$ \\
\hline AC FÍSICO & $2,32 \pm 0,87$ & $1,84 \pm 0,77$ & 2,756 & $0,008^{*}$ & $0,13303-0,84211$ \\
AC GLOBAL & $2,59 \pm 0,84$ & $2,38 \pm 0,91$ & 1,041 & 0,304 & $-0,19260-0,60474$ \\
\hline
\end{tabular}

$\mathrm{AC}=$ autoconceito; $\mathrm{cm}=$ centímetros. $\left({ }^{*}\right) \mathrm{p}<0,005$. 


\section{Falconi, C.A.; Santos, T. A., Figueira Junior, A. J., Neves, A. N., Andrade, E. L. ; Brandão, M. R.}

F.; Zanetti, M. C.

A tabela 4 apresenta os resultados dos testes de correlação entre os domínios do autoconceito físico e o autoconceito global, com as variáveis antropométricas e o percentual de gordura. Foram encontradas correlações negativas entre 0 autoconceito físico e a circunferência de cintura. $\mathrm{O}$ autoconceito global não apresentou correlação com nenhuma das variáveis avaliadas.

\section{Tabela 4.}

Teste de Correlação de Spearman entre as variáveis antropométricas e o autoconceito.

\begin{tabular}{lcc}
\hline \multicolumn{1}{c}{ Correlação } & $\boldsymbol{R}$ & $\mathbf{p}$ \\
\hline AC Físico X IMC & $-0,140$ & 0,162 \\
AC Físico X Percentual de gordura & 0,007 & 0,945 \\
AC Físico X Circunferência de cintura & $-0,266$ & $0,007^{*}$ \\
AC Global X IMC & $-0,082$ & 0,477 \\
AC Global X Percentual de gordura & $-0,029$ & 0,775 \\
AC Global X Circunferência de cintura & $-0,165$ & 0,099 \\
\hline
\end{tabular}

$\mathrm{AC}=$ autoconceito; IMC $=$ índice de massa corporal; $(*) \mathrm{p}<0,005$. Segundo Field (2009) a correlação teve um efeito pequeno quando $r \leq 0,299$, efeito médio $r \leq 0,499$ e grande $r \geq 0,50$.

\section{DISCUSSÃO}

O objetivo do presente estudo foi verificar a relação entre os índices antropométricos, circunferência de cintura, IMC e o percentual de gordura corporal com o autoconceito físico e global de adolescentes do sexo feminino. Para tal foram verificadas as diferenças entre os grupos levando-se em consideração a circunferência de cintura conforme os parâmetros de saúde preconizados pelo IDF (2007). Essa análise demonstrou que as adolescentes que apresentavam cintura $\geq 80 \mathrm{~cm}$ também tinham menores escores de autoconceito físico.

Ao correlacionar as variáveis investigadas, identificou-se uma correlação inversa com efeito pequeno $(\mathrm{r}=-0,266)$ e entre o autoconceito físico e a circunferência de cintura. Esses achados são reforçados pelos estudos de Mitchell et al. (2012) que identificou valores correlacionais próximos aos encontrados ao analisar a relação entre o autoconceito físico e circunferência de cintura em alunos do ensino fundamental $(\mathrm{r}=-0,260, \mathrm{p}<0,001)$. García-Sáchez et al. (2013) também encontraram correlação negativa entre essas variáveis ao estudar adolescentes espanhóis $(\mathrm{r}=-0,304 ; \mathrm{p}<0,05)$, mostrando que a circunferência de cintura elevada pode estar associada a um autoconceito físico menor.
Embora esse estudo não tenha encontrado correlação entre IMC e percentual de gordura corporal com o autoconceito das adolescentes, vários estudos encontraram resultados contrários, associando o menor autoconceito físico com maiores valores de IMC (Mitchell et al. 2012; Jelalian et al., 2011; Wallander et al., 2009; Adams e Bukowski, 2008; O'dea, 2006; Savoye et al., 2005). Um estudo longitudinal conduzido por O'dea (2006), acompanhou por 5 anos 2.379 meninas e mostrou que a condição de ter um maior IMC estava relacionado com um menor autoconceito físico e global, e que esse quadro não tendeu a mudanças com o avanço da idade, o que pode sugerir possíveis problemas que são refletidos na idade adulta. Além disso, outros estudos apontaram a associação do IMC mais alto com outras dimensões do autoconceito, principalmente na dimensão social (Strauss, 2000; Brown et al., 1998;).

Contudo, apesar de dois terços da amostra ter sido classificada como eutrófica com parâmetros de gordura corporal dentro da normalidade para essa população, podemos apontar que nesse estudo a circunferência de cintura elevada foi significativamente associada a avaliações mais pessimistas do autoconceito físico de adolescentes do sexo feminino $(p=0,008)$, mas sem significância no autoconceito global $(\mathrm{p}=0,304)$. 


\section{Medidas do corpo e autoconceito de adolescentes do sexo feminino}

O presente estudo possui algumas limitações, dentre elas o corte transversal que embora seja rápido e prático para observar fenômenos em determinada população não permite estabelecer a relação causaefeito. Nesse sentido, não conduz ao entendimento se o autoconceito físico mais baixo seja o causador da circunferência de cintura aumentada por associação de outros fatores, como por exemplo, a busca por dietas palatáveis como recompensas psicológicas levando ao aumento do tecido adiposo, ou se o aumento da circunferência de cintura causa uma avaliação negativa da aparência física associada ao feedback dos pares. Outrossim, não pode ser interpretado de forma generalizada, pois não foi uma amostra significativa de adolescentes e a escolha por conveniência não permite o entendimento de outros fatores, como estilo de vida e influencias sociais, que podem ser específicos desse grupo, como por exemplo maior instrução já que os alunos ingressam nessa escola por meio de avaliação de conhecimento (Vestibulinho). Contudo, há a necessidade de novos estudos que acompanhem tamanhos amostrais significativos para buscar relacionar causa-efeito e aumentar o poder estatístico dos resultados, que aborde mais variáveis relacionadas a importância hierárquica dos domínios do autoconceito do adolescente e inclua outras dimensões do autoconceito.

\section{CONCLUSÃO}

Conclui-se que por meio das análises das variáveis observadas, podemos apontar nesse estudo que a circunferência de cintura elevada foi significativamente associada a avaliações mais pessimistas do autoconceito físico de adolescentes do sexo feminino, mas sem significância no autoconceito global.

\section{APLICAÇÕES PRÁTICAS}

A baixa aptidão física provocada pela falta de atividades físicas e o comportamento alimentar contemporâneo com alta ingesta de produtos hipercalóricos pode trazer consequências metabólicas danosas para a saúde, principalmente a saúde cardiovascular dos adolescentes. O aumento do peso corporal, do percentual de gordura e da circunferência de cintura são alguns dos indicadores que podem prever riscos à saúde da população. $\mathrm{O}$ presente estudo pode apontar, que além dos problemas metabólicos amplamente citados na literatura, esses indicadores parecem estar associados mesmo que de forma pequena, com o autoconceito de jovens, sugerindo uma relação negativa, danosa ao bem-estar da jovem com circunferência de cintura aumentada. Dessa forma o estudo demostra que o aumento de indicadores antropométricos de sobrepeso, pode se relacionar com aspectos biopsicossociais de jovens adolescentes do sexo feminino.

\section{REFERÊNCIAS}

1. Adams, R. E.; Bukowski, W. M. Peer victimization as a predictor of depression and body mass index in obese and non-obese adolescentes. Journal of Child Psychology and Psychiatry, 49:8 (2008), pp 858-866.

2. Associação Brasileira para o Estudo da Obesidade e da Síndrome Metabólica. Mapa da obesidade 2016 [cited 2016 18/10/2016]. Available from: http://www.abeso.org.br/atitudesaudavel/mapa-obesidade

3. Bandeira D. R., Arteche A. X., Reppold CT. Escala de Autopercepção de Harter para adolescentes: um estudo de validação. Psicologia: teoria e pesquisa. Brasília Vol 24, n 3 (jul/set 2008), p 341-345. 2008.

4. Bee, H. A criança em desenvolvimento. $7^{\mathrm{a}}$ ed. Editora Artmed, 1996.

5. Brown, K. M., McMahon, R. P., Biro, F. M., Crawford, P., Schreiber, G. B., Similo, M. S. et al. (1998). Changes in self-esteem in black and white girls between the ages of 9 and 14 years. The NHLBI growth and health study. Journal of Adolescent Health, 23, 7-19.

6. Field, A. Descobrindo a Estatística usando o SPSS. Porto Alegre: ArtMed, 2009.

7. García-Sánchez, A. et al. Condición física, adiposidad y autoconcepto en adolescentes. Estudio piloto. Revista de Psicología del deporte, v. 22, n. 2, 2013.

8. Guerra PH, Farias Júnior J. C., Florindo A. A. Comportamento sedentário em crianças e adolescentes brasileiros: revisão sistemática. Revista de Saúde Pública. 2016;50. 


\section{Falconi, C.A.; Santos, T. A., Figueira Junior, A. J., Neves, A. N., Andrade, E. L. ; Brandão, M. R.}

F.; Zanetti, M. C.

9. Harter, S. The construction of the self: A developmental perspective. (1999). New York: Guilford Press.

10. International Diabetes Federation. Criteria of metabolic syndrome in children and adolescents, 20072016 [20/04/2016]. Available from: http://www.idf.org/metabolicsyndrome/children/criteria.

11. Jelalian, E.; Sato, A.; Hart, C. N. The effect of group-based weight-control intervention on adolescent psychosocial outcomes: Perceived peer rejection, social anxiety, and self-concept. Children's Health Care, v. 40, n. 3, p. 197-211, 2011.

12. Kvaavik E., Klepp K-I., Tell G. S., Meyer H. E., Batty G. D. Physical fitness and physical activity at age 13 years as predictors of cardiovascular disease risk factors at ages $15,25,33$, and 40 years: extended follow-up of the Oslo Youth Study. Pediatrics. 2009;123(1):e80-e6.

13. Lohman, T. G. Advances in body composition assessment. Champaign. Human Kinetics Publishers, 1992.

14. Matsudo, S. M., Actividad Física y Ejercicio em Salud y Enfermedad. $1^{\text {a }}$ Edição, Editora Mediterrâneo, Santiago, Chile, 2017. 455p.

15. Massuça, L.; Proença, J. A massa gorda de risco afeta a capacidade aeróbica de jovens adolescentes. Revista Brasileira de Medicina do Esporte - Vol. 19, № 6 - Nov/Dez, 2013.

16. Mitchell, N. G., Moore, J. B., Bibeau, W. S. y Rudasill, K. M. (2012). Cardiovascular Fitness Moderates the Relations Between Estimates of Obesity and Physical Self-Perceptions in Rural Elementary School Students. Journal of Physical Activity \& Health, 9(2), 288-294.

17. O’Dea, J. A. (2006). Self-concept., self-esteem and body weight in adolescent females - A threeyear longitudinal study. Journal of Health Psychology, 11(4). 599-611.

18. Pereira, T. A.; Araújo Bergmann, M. L.; Bergmann, G. G.; Fatores associados a baixa aptidão física em adolescentes. Revista Brasileira de Medicina do Esporte - Vol. 22, No 3 Mai/Jun, 2016.
19. Santana C. C. A., Andrade L. P., Gama V. D., Mota J., Prado W. L. Associação entre estado nutricional e aptidão física relacionada à saúde em crianças. Journal of Physical Education. 2013;24(3):433-41.

20. Santana, V. H. Avaliação do autoconceito aplicada à atividade física. 2003. 113 fls. Dissertação (Mestrado em Educação Física) Faculdade de Educação Física, Universidade Estadual de Campinas, São Paulo, 2003.

21. Savoye, M., Berry, D., Dziura, J., Shaw, M., Serrecchia, J. B., Barbetta, G. y Caprio, S. (2005). Anthropometric and psychosocial changes in obese adolescents enrolled in a Weight Management Program. Journal of the American Dietetic Association, 105(3), 364-370.

22. Serassuelo Junior, $H$. et al. The self-concept perception and its influence on motor performances of childrens and adolescents. Revista da Educação Física/UEM, v. 23, n. 1, p. 15-23, 2012.

23. Slaughter, M. H.; Lohman, T. G.; Boileau, R. A.; Horswill, C. A.; Stillman, R. J.; Vanloan, M. D.; Bemben, D. A. Skinfold equations for estimation of body fatness in children and youth. Human Biology. 1988; 60: 709-723.

24. Sociedade Brasileira de Hipertensao. I Diretriz Brasileira de Diagnóstico e Tratamento da Síndrome Metabólica. Arquivos Brasileiros de Cardiologia. 2005;84(Suplemento I).

25. Strauss, R. S. (2000). Childhood obesity and selfesteem. Pediatrics, 105(1). Available at http//www.pediatrics.org/cgi/content/full/105/1/e 15.

26. Villa Sánches A., Escribano E. A. Medição do autoconceito. Bauru, SP: Editora da Universidade do Sagrado Coração; 1999.

27. Wallander, J. L., Taylor, W. C., Grunbaum, J. A., Franklin, F. A., Harrison, G. G., Kelder, S. H. y Schuster, M. A. (2009). Weight Status, Quality of Life, and Self-concept in African American, Hispanic, and White Fifth-grade Children. Obesity, 17 (7), 1363-1368.

28. Wieting J. M., Cause and effect in childhood obesity: solutions for a national epidemic. The 
Medidas do corpo e autoconceito de adolescentes do sexo feminino

Journal of the American Osteopathic Association. 2008;108(10):545-52.

29. World Health Organization - WHO (2006). CHILD GROWTH STANDARDS: METHODS AND DEVELOPMENT: LENGTH/HEIGHTFOR-AGE, WEIGHT-FOR-AGE, WEIGHTFOR-LENGTH, WEIGHT-FOR-HEIGHT AND BODY MASS INDEX-FOR-AGE. GENEVA: World Health Organization. 